\title{
Magnesium Oxide/Carbon Nanofiber Nanocomposite Modified Electrode for Electrochemical Hemoglobin Sensor
}

\author{
Hamza Abdalla Yones, Lin Zhu, Bo Shao, Siyue Zhang, Hui Xie, Xiaoqing Li, Wei Sun* \\ Key Laboratory of Water Pollution Treatment and Resource Reuse of Hainan Province, Key \\ Laboratory of Functional Materials and Photoelectrochemistry of Haikou, College of Chemistry and \\ Chemical Engineering, Hainan Normal University, Haikou 571158, P. R. China \\ *E-mail: sunwei@hainnu.edu.cn
}

doi: $10.20964 / 2021.02 .37$

Received: 3 October 2020 / Accepted: 26 November 2020 / Published: 31 December 2020

\begin{abstract}
$\mathrm{MgO}$ decorated carbon nanofiber (MgO@CNFs) nanocomposite was prepared by electrospinning and carbonization method. Then the nanocomposite was casted on carbon ionic liquid electrode (CILE) with the following immobilization of hemoglobin $(\mathrm{Hb})$ by Nafion polymer film. UV-Vis spectroscopic results showed that $\mathrm{Hb}$ maintained its original structure without changing after mixing with nanocomposite. The resultant $\mathrm{MgO} @ \mathrm{CNFs}$ modified electrode provided a favorable microenvironment for $\mathrm{Hb}$ to realize electrochemistry and the bioelectrochemical properties of $\mathrm{Hb}$ were studied in details. The results showed that $\mathrm{MgO} @ \mathrm{CNFs}$ nanocomposite on electrode improved Hb loading amount with its bioactivity maintained and electron transfer rate fasten, which was attributed to big specific interface area, excellent biocompatibility and high conductivity. Nafion/Hb/MgO@CNFs/CILE also displayed high electrocatalytic activity to the electroreduction of trichloroacetic acid. This study provided a novel way for the fabrication of the nanostructured biosurfaces for electrochemical biosensors.
\end{abstract}

Keywords: $\mathrm{MgO}$ decorated carbon nanofiber; Hemoglobin; Biosensor; Direct electrochemistry; Electrocatalysis

\section{FULL TEXT}

(C) 2021 The Authors. Published by ESG (www.electrochemsci.org). This article is an open access article distributed under the terms and conditions of the Creative Commons Attribution license (http://creativecommons.org/licenses/by/4.0/). 